UVX 2012, $02001(2013)$

DOI: $10.1051 /$ uvx/201302001

(C) Owned by the authors, published by EDP Sciences, 2013

\title{
Analysis by Coupled-Mode Theory of X-ray Multilayer Gratings
}

\author{
J.-M. André, P. Jonnard and K. Le Guen \\ Laboratoire de Chimie Physique-Matière et Rayonnement, Université Pierre et Marie Curie, \\ CNRS UMR 7614, 11 rue Pierre et Marie Curie, 75231 Paris Cedex 05, France
}

\begin{abstract}
Multilayer gratings developed since the end of the ninethies are now used for various purposes in x-uv optics: polychromator, narrow bandpass monochromators... We have implemented the Coupled-Mode Theory (CMT) to calculate the performances of these devices (efficiencies, bandwidths...) especially for quasi-lamellar gratings with different shapes of the multilayer pitch. We have fabricated such gratings and compared CMT calculations with measurements obtained at the BEAR beamline of ELETTRA.
\end{abstract}

\section{INTRODUCTION}

Structures with a spatially modulated polarizability provide new solutions to problems of optics and spectroscopy. The multilayer gratings (MGs), consisting of a periodic stack of bilayers with nanometric thickness laterally etched according to a periodic pattern, belong to this class of 2D photonic crystals that has opened up new ways for x-ray optics. Thus lamellar multilayer gratings (LMGs) can be used as efficient polychromators [1] and can also be implemented as improved monochromators with respect to mere multilayer Bragg mirrors for the soft-x-ray domain [2-8].

Calculations of the performances (efficiencies, bandwidths, ...) of these devices have been initially done in the framework of the "rigorous" modal theories which give rise to time-consuming computations and tricky numerical problems [9-11]. Among the latter are the numerical instabilities [12,13] and the mathematical difficulties in solving the transcendental equations to find the modal wavenumbers $[14,15]$. The level of difficulty is increasing as soon as the profile of the pattern is not lamellar, that is when the profile of the multilayer pitch varies with the depth. To overcome these problems, it has been recently proposed to appeal to the well-know coupled-waves approach; this one has been successfully performed for the lamellar MGs [16]. In this paper we adopt this approach for two different scopes:

- to get more physical insight in the so-called single-order regime of the LMGs;

- to study the influence of the profile of the multilayer pitch on the diffraction efficiency.

This latter point is important from the practical point of view; indeed recent measurements of the efficiency at the zero ${ }^{\text {th }}$ order of a Mo/ $\mathrm{B}_{4} \mathrm{C} \mathrm{MG}$ [17] have shown that the absolute peak efficiency is smaller and that the bandwidth is notably larger than the expected ones for a perfect LMG as given by the modal theory. One can suspect that these discrepancies can be attributed, at least in part, to a departure of the multilayer pitch from the rectangular shape.

\section{DESCRIPTION OF THE ETCHED MULTILAYER}

We consider the diffraction by an multilayer etched following the profile of a grating whose scheme is given in Figure 1. It consists of a multilayer stack built up with $\mathrm{N}$ bilayers of thickness $d$; each bilayer is

This is an Open Access article distributed under the terms of the Creative Commons Attribution License 2.0, which permits unrestricted use, distribution, and reproduction in any medium, provided the original work is properly cited. 


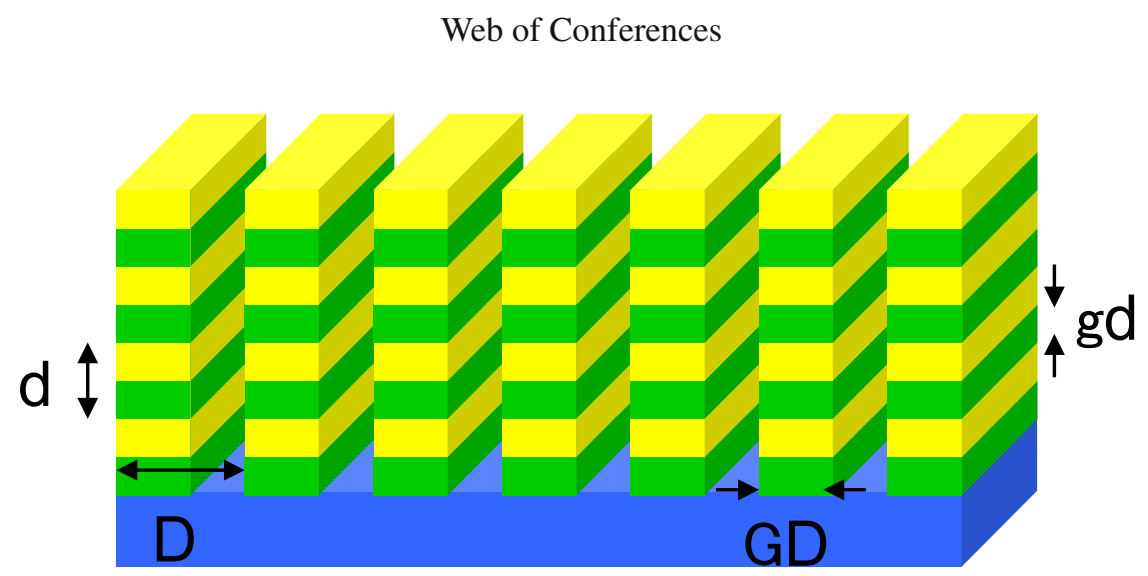

Figure 1. Scheme of the etched multilayer. D is the period of the multilayer. D is the period of the grating.

made up with a heavy material $l$ of thickness $\gamma d$ and polarizability $\chi_{1}$, and a light material 2 of thickness $(1-\gamma) d$ and polarizability $\chi_{2}$. We consider abrupt interfaces so that the polarizability $\chi(z)$ of the bilayer is given by:

$$
\chi(z)=\chi_{1}+u(z)\left(\chi_{2}-\chi_{1}\right)
$$

with

$$
u(z)=\gamma+\sum_{t \neq 0} u_{t} \exp \left(\frac{2 i \pi t z}{d}\right)
$$

and

$$
u_{t}=\frac{(1-\exp (-2 i \pi t \gamma))}{2 i \pi t} .
$$

The stack is etched to obtain a grating of period $\mathrm{D}$ with multilayer bar of width $\Gamma(z) D$ at the depth $z$. Consequently the profile of the grating along the $x$-axis is given by:

$$
U(x)=\Gamma_{0}(z)+\sum_{t \neq 0} \frac{\left(1-\exp \left(-2 i \pi t \Gamma_{0}(z)\right)\right)}{2 i \pi t} \exp \left(\frac{2 i \pi t x}{D}\right) .
$$

Then the function $\Gamma_{0}(z)$ allows us to deal with a grating whose bar profile is arbitrary. In the usual case of a laminar grating, one has $\Gamma_{0}(z)=\Gamma_{0}$. The $2 \mathrm{D}$ polarizability of the grating under consideration is given by:

$$
\chi(x, z)=\chi(z) U(x) .
$$

\section{EQUATIONS OF COUPLED-MODE THEORY}

The electric field $E(x, z)$ in the structure obeys the 2D propagation equation for the $s$-polarization case:

$$
\nabla^{2} E(x, z)+k^{2}(1-\chi(x, z)) E(x, z)=0
$$

solution of which can be expanded as:

$$
E(x, z)=\sum_{n=-\infty}^{+\infty} \mathcal{E}_{n}(z) \exp \left(i k_{\| n} x\right)
$$

with

$$
k_{\| n}=k \cos \theta+\frac{2 \pi n}{D}
$$

where $k$ is the wavenumber in vacuum and $\theta$ the grazing angle of the incident radiation. 
Following the principle of the Coupled-Mode Theory CMT, the $\mathrm{n}^{\text {th }}$ order of the field $\mathcal{E}_{n}(z)$ is searched as a superposition of two counter-propagating waves whose respective amplitudes $F$ and $B$ vary with $z$, that is:

$$
\mathcal{E}_{n}(z)=F_{n}(z) \exp \left(i k_{\perp n} z\right)+B_{n}(z) \exp \left(-i k_{\perp n} z\right)
$$

where

$$
k_{\perp n}=\sqrt{k^{2}-k_{\| n}^{2}}
$$

For the sake of uniqueness, the additional condition is required:

$$
\dot{F}_{n}(z) \exp \left(i k_{\perp n} z\right)+\dot{B}_{n}(z) \exp \left(-i k_{\perp n} z\right)=0 .
$$

By substituting Eqs. (9) and (11) into Eqs. (6) and (7), it follows that the amplitudes satisfy the system of differential equations written in matrix form:

$$
\frac{d A(z)}{d z}=\bar{D}(z) A(z)
$$

where A(z) stands for the column matrix of the amplitudes

$$
\left(\begin{array}{c}
\vdots \\
F_{-n}(z) \\
B_{-n}(z) \\
\vdots \\
F_{n}(z) \\
B_{n}(z) \\
\vdots
\end{array}\right)
$$

and $\bar{D}(z)$ is the square matrix the components of which are:

$$
D_{n m}(z)=-\frac{i k^{2}}{2 k_{\perp n}} \chi(z) U_{n-m}(z) \exp \left[+i\left(k_{\perp m}-k_{\perp n}\right)\right]
$$

in the rows corresponding to the terms $\frac{d F_{n}(z)}{d z}$ and the columns affecting the terms $F_{n}(z)$,

$$
D_{n m}(z)=-\frac{i k^{2}}{2 k_{\perp n}} \chi(z) U_{n-m}(z) \exp \left[-i\left(k_{\perp m}+k_{\perp n}\right)\right]
$$

in the rows corresponding to the terms $\frac{d F_{n}(z)}{d z}$ and the columns affecting the terms $B_{n}(z)$,

$$
D_{n m}(z)=\frac{i k^{2}}{2 k_{\perp n}} \chi(z) U_{n-m}(z) \exp \left[+i\left(k_{\perp m}+k_{\perp n}\right)\right]
$$

in the rows corresponding to the terms $\frac{d B_{n}(z)}{d z}$ and the columns affecting the terms $F_{n}(z)$,

$$
D_{n m}(z)=\frac{i k^{2}}{2 k_{\perp n}} \chi(z) U_{n-m}(z) \exp \left[-i\left(k_{\perp m}-k_{\perp n}\right)\right]
$$

in the rows corresponding to the terms $\frac{d B_{n}(z)}{d z}$ and the columns affecting the terms $B_{n}(z)$.

In this study, we assume that there is only one plane wave incident on the grating, which gives the following boundary conditions:

$$
F_{n}(0)=\delta_{n, 0} \quad \text { and } \quad B_{n}(L)=0
$$

Let us note that this theory can be applied to the $p$-polarization case provided that one replaces in Eq. (6) the electric field by the modified magnetic field $\tilde{H}(x, z)=H(x, z) / \sqrt{1-\chi(x, z)}$ and the polarizability 


\section{Web of Conferences}

$\chi(x, z)$ by the following modified polarizability [16]:

$$
\tilde{\chi}(x, z)=\chi(x, z)+\frac{1}{2 k^{2}} \frac{\nabla^{2} \chi(x, z)}{1-\chi(x, z)}-\frac{3}{4 k^{2}} \frac{[\vec{\nabla} \chi(x, z)]^{2}}{(1-\chi(x, z))^{2}} .
$$

\section{SINGLE-ORDER REGIME ANALYSIS}

In this operating regime, the incident plane wave only excites one single grating order [7]. Let us assume this order to be the zero ${ }^{\text {th }}$ one; this case corresponds to the specular reflection of interest in the application of the narrow bandwidth monochromator. In this case, only the field

$$
\mathcal{E}_{0}(z)=F_{0}(z) \exp \left(i k_{\perp 0} z\right)+B_{0}(z) \exp \left(-i k_{\perp 0} z\right)
$$

is relevant and the matrix $\bar{D}(z)$ reduces to a $2 \times 2$ matrix; the system Eq. (7) is the system of two coupled equations:

$$
\frac{d}{d z}\left(\begin{array}{l}
F_{0}(z) \\
B_{0}(z)
\end{array}\right)=\left(\begin{array}{cc}
\frac{-i k^{2}}{2 k_{\perp 0}} \Gamma(z) \chi(z) & \frac{-i k^{2}}{2 k_{\perp 0}} \Gamma(z) \chi(z) \exp \left(-2 i k_{\perp 0} z\right) \\
\frac{i k^{2}}{2 k_{\perp 0}} \Gamma(z) \chi(z) \exp \left(+2 i k_{\perp 0} z\right) & \frac{i k^{2}}{2 k_{\perp 0}} \Gamma(z) \chi(z)
\end{array}\right)\left(\begin{array}{l}
F_{0}(z) \\
B_{0}(z)
\end{array}\right) .
$$

\subsection{Case of a laminar multilayer grating near Bragg resonance}

Let us consider the particular case of a laminar multilayer grating near the $n^{\text {th }}$ Bragg resonance; in this case $\Gamma(z)$ does not vary versus $\mathrm{z}$ and takes the constant value $\Gamma_{0}$. The matrix $\bar{D}(z)$ becomes:

$$
\bar{D}(z)=\left(\begin{array}{cc}
\frac{-i k^{2}}{2 k_{\perp 0}} \Gamma_{0} \bar{\chi} & \frac{-i k^{2}}{2 k_{\perp 0}} \Gamma_{0} \Delta \chi u_{n} \exp (2 i \kappa z) \\
\frac{i k^{2}}{2 k_{\perp 0}} \Gamma_{0} \Delta \chi u_{-n} \exp (-2 i \kappa z) & \frac{i k^{2}}{2 k_{\perp 0}} \Gamma_{0} \bar{\chi}
\end{array}\right)
$$

with

$$
\kappa=\frac{\pi n}{d}-k_{\perp 0}
$$

The Bragg condition $k_{\perp 0} \simeq \frac{\pi n}{d}$, that is $\kappa \approx 0$, is nearly satisfied close to the $n^{\text {th }}$ Bragg resonance. In order to obtain coupled differential equations with constant coefficients, it is convenient to introduce the associated amplitude:

$$
\hat{F}_{0}(z)=F_{0}(z) \exp (-i \kappa z)
$$

and

$$
\hat{B}_{0}(z)=B_{0}(z) \exp (+i \kappa z)
$$

so that

$$
\frac{d}{d z}\left(\begin{array}{l}
\hat{F}_{0}(z) \\
\hat{B}_{0}(z)
\end{array}\right)=\bar{M}\left(\begin{array}{l}
\hat{F}_{0}(z) \\
\hat{B}_{0}(z)
\end{array}\right)
$$

with

$$
\bar{M}=\left(\begin{array}{ll}
\frac{-i k^{2}}{2 k_{\perp 0}} \Gamma_{0} \bar{\chi}-i \kappa & \frac{-i k^{2}}{2 k_{\perp 0}} \Gamma_{0} \Delta \chi u_{n} \\
\frac{i k^{2}}{2 k_{\perp 0}} \Gamma_{0} \Delta \chi u_{-n} & \frac{i k^{2}}{2 k_{\perp 0}} \Gamma_{0} \bar{\chi}+i \kappa
\end{array}\right)=\left(\begin{array}{cc}
-\beta & \mathrm{K}_{1} \\
\mathrm{~K}_{2} & \beta
\end{array}\right) .
$$




\section{UVX 2012}

\subsection{Eigenstate solutions of the CM equations}

The solution of the above equations can be obtained by substituting:

$$
\left(\begin{array}{l}
\hat{F}_{0}(z) \\
\hat{B}_{0}(z)
\end{array}\right)=\left(\begin{array}{l}
\hat{F}_{0} \\
\hat{B}_{0}
\end{array}\right) \exp (i q z)
$$

into Eq. (23), to get the following eigenvalue problem:

$$
(\bar{M}-q \bar{I})\left(\begin{array}{c}
\hat{F}_{0} \\
\hat{B}_{0}
\end{array}\right)=\overline{0} .
$$

There are two eigenvalues:

$$
q^{ \pm}= \pm q= \pm \sqrt{\mathrm{K}_{1} \mathrm{~K}_{2}+\beta^{2}}= \pm \sqrt{\left(\frac{i k^{2}}{2 k_{\perp 0}} \Gamma_{0} \bar{\chi}+i \kappa\right)^{2}-\left(\frac{k^{2}}{2 k_{\perp 0}} \Gamma_{0} \Delta \chi\right)^{2} u_{n-1} u_{n}}
$$

corresponding to the two eigenvectors:

$$
\overrightarrow{V^{+}}=\left(\begin{array}{c}
\mathrm{K}_{1} \\
q-\beta
\end{array}\right) \quad \text { and } \quad \overrightarrow{V^{-}}=\left(\begin{array}{c}
\mathrm{K}_{1} \\
-q-\beta
\end{array}\right)
$$

from which, one can construct the eigenmatrix $\bar{V}=\left[\overrightarrow{V^{+}}, \overrightarrow{V^{-}}\right]$. The general solution is a linear combination of eigensolutions:

$$
\left(\begin{array}{l}
\hat{F}_{0}(z) \\
\hat{B}_{0}(z)
\end{array}\right)=\bar{V}\left(\begin{array}{cc}
\exp (+i q z) & 0 \\
0 & \exp (-i q z)
\end{array}\right)\left(\begin{array}{l}
F \\
B
\end{array}\right) .
$$

Evaluating Eq. (30) at $z=0$ give $\mathrm{F}$ and $\mathrm{B}$ :

$$
\left(\begin{array}{l}
F \\
B
\end{array}\right)=\bar{V}^{-1}\left(\begin{array}{l}
\hat{F}_{0}(0) \\
\hat{B}_{0}(0)
\end{array}\right)
$$

Substituting Eq. (31) into Eq. (30) leads to:

$$
\left(\begin{array}{l}
\hat{F}_{0}(z) \\
\hat{B}_{0}(z)
\end{array}\right)=\bar{V}\left(\begin{array}{cc}
\exp (+i q z) & 0 \\
0 & \exp (-i q z)
\end{array}\right) \bar{V}^{-1}\left(\begin{array}{l}
\hat{F}_{0}(0) \\
\hat{B}_{0}(0)
\end{array}\right)=S(z)\left(\begin{array}{c}
\hat{F}_{0}(0) \\
\hat{B}_{0}(0)
\end{array}\right) .
$$

where $S(z)$ is the transfer matrix:

$$
S(z)=\left(\begin{array}{cc}
\cos (q z)+i \frac{\beta}{q} \sin (q z) & i \frac{\mathrm{K}_{2}}{q} \sin (q z) \\
i \frac{\mathrm{K}_{1}}{q} \sin (q z) & \cos (q z)-i \frac{\beta}{q} \sin (q z)
\end{array}\right) .
$$

Since only one plane wave is incident at $z=0$ from the incident medium $\hat{F}_{0}(0)=1, \hat{B}_{0}(0)=0$, it comes that:

$$
\hat{B}_{0}(z)=\left|\frac{\mathrm{K}_{1} \sin (q z)}{q}\right|^{2} .
$$

This analysis shows that energy is transferred through the coupling provided by the grating between the forward propagating wave and the backward travelling wave; according to Eq. (34), the transfer will be maximum for:

$$
q z=(2 s+1) \frac{\pi}{2}, s \text { integer. }
$$

Indeed since the coupling is asynchronous (the terms in the first diagonal of $\bar{M}$ are not equal), the transfer is never complete. Taking into account the boundary conditions considered in this study (reflection and transmission at the boundaries $=0$ and $z=L$ are neglected), one can deduce the reflection $r$ and 


\section{Web of Conferences}

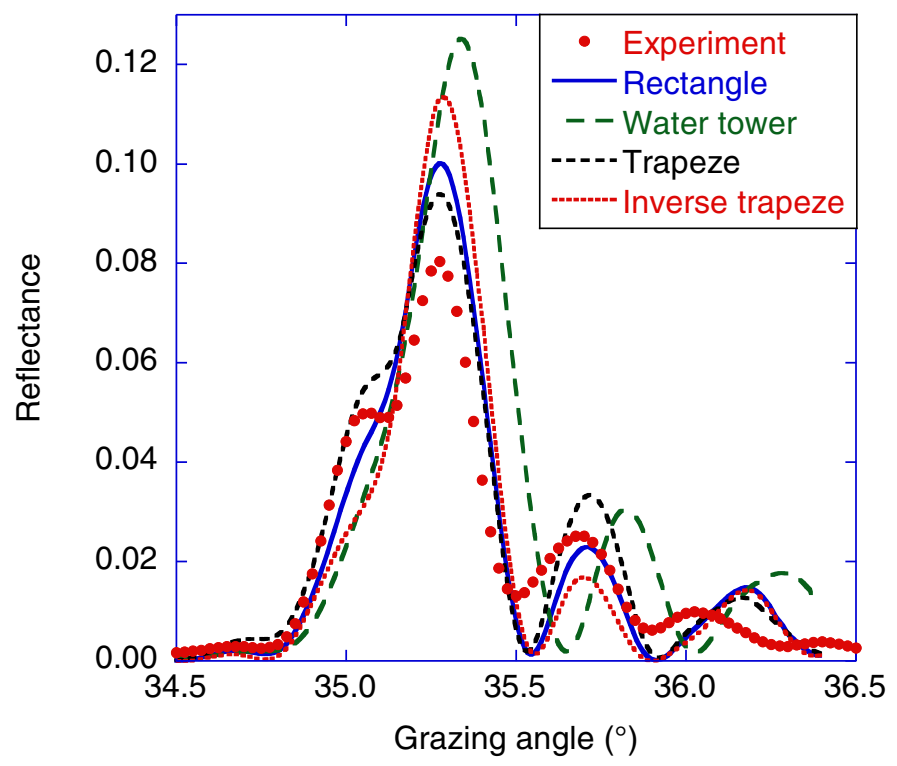

Figure 2. Reflectivity curves in the B K emission range: comparison between experiment and calculations performed for different profiles of the multilayer bar.

transmission $t$ coefficients:

$$
\begin{gathered}
r \equiv \frac{B_{0}(0)}{F_{0}(0)}=-\frac{S_{21}(L)}{S_{22}(L)}=\frac{-i \mathrm{~K}_{2} \sin (q L)}{q \cos (q L)-i \beta \sin (q L)} \\
t \equiv \frac{F_{0}(L)}{F_{0}(0)}=\frac{\operatorname{det}[S(L)]}{S_{22}(L)}=\frac{1}{S_{22}(L)}=\frac{q}{q \cos (q L)-i \beta \sin (q L)} .
\end{gathered}
$$

Let us mention that from Eq. (36) it is possible to find useful formula (such a generalized Bragg law for laminar multilayer grating) and optimization criteria; the reader can refer to the paper by Kozhevnikov et al. [16] for further information. It is interesting to note that the above formalism is similar to the one of the CMT of optical waveguide couplers, so that one gets some insights in the physics of the laminar multilayer grating in single-order regime from studies about coupled waveguides in contra-directional coupling $[18,19]$.

At this stage it is interesting to consider the particular case of lossless media. In this case,

$$
\mathrm{K}_{1}=K \quad \text { and } \quad \mathrm{K}_{2}=-K^{*}
$$

and the quantity $q$ can be written:

$$
q=\sqrt{-|K|^{2}+\beta^{2}}
$$

It can be purely imaginary if the condition:

$$
|K|>\beta
$$

is satisfied.

The present CMT is applied to study the influence of the profile of the multilayer bar onto the shape of the diffraction pattern at the zero ${ }^{\text {th }}$ order of a $\mathrm{MG}$ intended as narrow bandwidth monochomator for the spectral region around $180 \mathrm{eV}$ corresponding to the B-K emission band. The MG consists of $200 \mathrm{Mo} / \mathrm{B}_{4} \mathrm{C}$ bilayers etched with a period Dequal to $1 \mu \mathrm{m}$. The multilayer period $d$ and the ratio $\gamma$ are equal to $6.2 \mathrm{~nm}$ and 0.35 respectively. Thus the total thickness $L$ of the multilayer bar is $1240 \mathrm{~nm}$. Such a 
grating has been designed by our team. The diffraction efficiency at the zero ${ }^{\text {th }}$ order of a similar grating fabricated in the same conditions has been measured at $182 \mathrm{eV}$ versus the glancing angle at the BEAR beamline of the ELETTRA synchrotron facility [17].

To calculate the diffraction of the MG we have numerically solved the system of differential equations Eq. (12) with the boundary conditions given by Eq. (16) using a Runge-Kutta method; eleven orders have been retained in the calculation. Figure 2 show the zero ${ }^{\text {th }}$ order efficiency versus the glancing for different profiles listed below and in the range of the B K emission:

- rectangular shape (lamellar grating): $\Gamma_{0}(z)=0.18$;

- trapezoidal shape with the summit less than the basis: $\Gamma_{0}(z)=0.18+10^{-4} z$;

- trapezoidal shape with the summit larger than the basis: $\Gamma_{0}(z)=0.18-10^{-4} z$;

- "water tower" shape: $\Gamma_{0}(z)=0.285-2.410^{-4}$ zif0 $<\mathrm{z}<600$ and $\Gamma_{0}(z)=0.143$ if $z>600$.

The comparison of the experimental and calculated reflectivity curves shows that the reflectance is overestimated in the calculation. This is probably due to the interfacial roughness, not taken into account in the calculation. Now comparing the shape of the curves and the position of the main and secondary maxima, it can be seen that the water tower shape does not enable to reproduce the experiment. A shape intermediate between those of the rectangle and trapeze would lead to an appropriate description of the experimental curve. This should be confirmed by scanning electron microscopy. But this would require the destruction of the sample in order to observe its cross section.

\section{References}

[1] K. Krastev, F. LeGuern, K. Coat, R. Barchewitz, J.-M. André, M. F. Ravet, E. Cambril, F. Rousseaux, and P. Davi, Nucl. Instrum. Methods Phys. Res. Sect. A 368, 533-542 (1996)

[2] A.-E. Sammar, J.-M. André, M. Ouahabi, B. Pardo, and R. Barchewitz, C.R. Acad. Sci., Ser. 2, 316, 1055-1060 (1993)

[3] A. Sammar, K. Krastev, J.-M. André, R. Barchewitz, and R. Rivoira, Rev. Sci. Instrum. 68, 2969-2972 (1997)

[4] J.-M. André, R. Benbalagh, R. Barchewitz, M.-F. Ravet, A. Raynal, F. Delmotte, F. Bridou, G. Julié, A. Bosseboeuf, R. Laval, G. Soullié, C. Rémond, and M. Fialin, Appl. Opt. 41, 239244 (2002)

[5] R. Benbalagh, J.-M. André, R. Barchewitz, P. Jonnard, G. Julié, L. Mollard, G. Rolland, C. Rémond, P. Troussel, R. Marmoret, and E.O. Filatova, Nucl. Instrum. Methods Phys. Res. Sect. A 541, 590-597 (2005)

[6] P. Jonnard, K. Le Guen, and J.-M. André, X-Ray Spectrom. 38, 117-120 (2009)

[7] I.V. Kozhevnikov, R. van der Meer, H.M.J. Bastiaens, K.-J. Boller, and F. Bijkerk, Opt. Express 18, 16234-16242 (2010)

[8] R. van der Meer, B. Krishnan, I.V. Kozhevnikov, M.J. De Boer, B. Vratzov, H.M.J. Bastiaens, J. Huskens, W.G. van der Wiel, P.E. Hegeman, G.C.S. Brons, K.-J. Boller, and F. Bijkerk, Proc. SPIE 8139, 81390Q (2011)

[9] A. Sammar, J.-M. André, and B. Pardo, Opt. Commun. 86, 245-254 (1991)

[10] A.I. Erko, B. Vidal, P. Vincent, Y.A. Agafonov, V.V. Martynov, D.V. Roschupkin, and M. Brunel, Nucl. Instrum. Methods Phys. Res. Sect. A 333, 599-606 (1993)

[11] L. Li, J. Opt. Soc. Am. A 10, 2581-2591 (1993)

[12] F. Montiel and M. Nevière, J. Opt. Soc. Am. A 11, 3241-3250 (1994)

[13] K. Krastev, J.-M. André, and R. Barchewitz, J. Opt. Soc. Am. A 13, 2027-2033 (1996)

[14] L. Botten, M. Craig, R. McPhedran, J. Adams, and J. Andrewartha, Opt. Acta 28, 1087-1102 (1981) 


\section{Web of Conferences}

[15] G. Tayeb and R. Petit, Opt. Acta 31, 1361-1365 (1984)

[16] I.V. Kozhevnikov, R. van der Meer, H.M.J. Bastiaens, K.-J. Boller, and F. Bijkerk, Opt. Express 19, 9172-9184 (2011)

[17] P. Jonnard, K. Le Guen, J.-M. André, J.-R. Coudevylle, and N. Isac, X-Ray Spectrometry (2012)

[18] P. Yeh and H.F. Taylor, Appl. Opt. 19, 2848-2855 (1980)

[19] W.-P. Huang, J. Opt. Soc. Am. A 11, 963-983 (1994) 